\title{
Robotic Competition Teams: Assessing the Experiential Education Value of Participation
}

\section{Mr. Andrew Jones, North Dakota State University}

Andrew Jones received a master's degree in Software Engineering from North Dakota State University (NDSU) in 2016. He is currently a Software Engineering Ph.D. student studying artificial intelligence and robotics. He is currently a teaching assistant and research assistant, and has served as the team lead for autonomous robot development competitions, such as the IGVC.

\section{Dr. Jeremy Straub, North Dakota State University}

Jeremy Straub is the Associate Director of the NDSU Institute for Cyber Security Education and Research and an Assistant Professor in the Department of Computer Science at the North Dakota State University. He holds a Ph.D. in Scientific Computing, an M.S. and an M.B.A. and has published over 40 journal articles and over 120 full conference papers, in addition to making numerous other conference presentations. Straub's research spans the gauntlet between technology, commercialization and technology policy. In particular, his research has recently focused on cybersecurity topics including intrusion detection and forensics, robotic command and control, aerospace command and 3D printing quality assurance. Straub is a member of Sigma Xi, SPIE, the AIAA and several other technical societies, he has also served as a track or session chair for numerous conferences. 


\section{Robotic Competition Teams: Assessing the Experiential Education Value of Participation}

\section{Introduction}

Competitions and prizes can contribute to innovative changes by influencing society or specific communities and individuals. According to Dias et al. [1], the potential societal benefits of competitions include identifying excellence, influencing public perception for a specific domain, focusing communities on specific problems and mobilizing new talent, strengthening problemsolving communities by educating individuals.

This research paper considers the value of robotics competitions from the perspective of their experiential education value. Each year, thousands of students across the United States and around the world participate in a wide variety of robotics competitions. It is generally recognized that student participants find these experiences highly enjoyable and have the opportunity to gain and demonstrate skills in a variety of areas directly and peripherally related to the project. However, despite the prevalence of these activities, insufficient research has been performed to characterize their specific value and the sources that it comes from.

This paper presents initial work on the characterization of the value of participation in robotics competition teams. Modified versions of surveys, initially designed and validated for (primarily undergraduate) research project participation, are used to collect information about student perception of the magnitude and attribution of gains in multiple areas. These surveys use questions from the Undergraduate Research Student Self-Assessment (URSSA) instrument [2] as well as questions initially designed to focus on particular activities and customized to focus on the specific values provided by robotic competition program participation.

This paper is organized as follows. First, background research in related and relevant topic areas are outlined. Second, the survey is discussed, and results presented. Third, the results from the survey are analyzed. Finally, the paper concludes with a discussion about planned future work in this area.

\section{Background}

In this section, work in related topics are discussed. First, related work in robotics competitions and education is outlined. Then, the benefits of problem-based learning are analyzed.

\subsection{Robotics Competitions and Education}

For robotics education, Zdešar et al. [3] found that students typically appreciate a balance between theory and practice. In this regard, the use of competitions could provide an excellent means of practice, which could be augmented with theory through the aid of a competition mentor or advisor. Moreover, a study done in 2002 by Ahlgren and Verner [4] showed that robotic contests could lead to considerable progress in theoretical and practical areas for students, both at the K-12 and university levels. 
The level of engagement and interest observed in students participating in robotics projects is also of note. Merkouris et al. [5] found that students were more engaged by programming robots than they were for programming applications for a desktop computer. Remote education and training are also possible through the simulation and programming of robots. An example of this is the Robotic Programming Network (RPN), which is an initiative that involves writing ROS code in an Internet browser and running it on a remote robot [6].

\subsection{Problem based learning}

According to Savery [7], problem based learning (PBL) is an instructional (and curricular) learner-centered approach that empowers learners to conduct research, integrate theory and practice, and apply knowledge and skills to develop a viable solution to a defined problem. In this approach, students have the responsibility for their own learning. In effect, this can provide an incentive for students to become more intrinsically motivated to learn, and potentially develop or enhance self-directed, lifelong learning skills [8]. Moreover, the problem is often an integration of a wide range of disciplines or subjects [7]. Thus, collaboration is essential, with students potentially becoming more effective collaborators as a result.

Robotics is an especially effective medium for problem-based learning for many reasons. According to Ahlgren et al. [9], these include: students become involved in self-directed learning, interdisciplinary design, teamwork, professional communication, technical invention, and research. Another reason is that intensive practice in solving diverse mental and physical tasks in the robotics medium can promote development of student intelligence and creativity [9].

\section{Robot Competitions}

There are a number of robot competitions that students in this study participated in. One example is the Intelligent Ground Vehicle Competition (IGVC), which involves building and programming a fully autonomous unmanned ground robotic vehicle. The vehicle must negotiate around an outdoor obstacle course under a prescribed time, remaining within the lane, and avoiding the obstacles on the course.

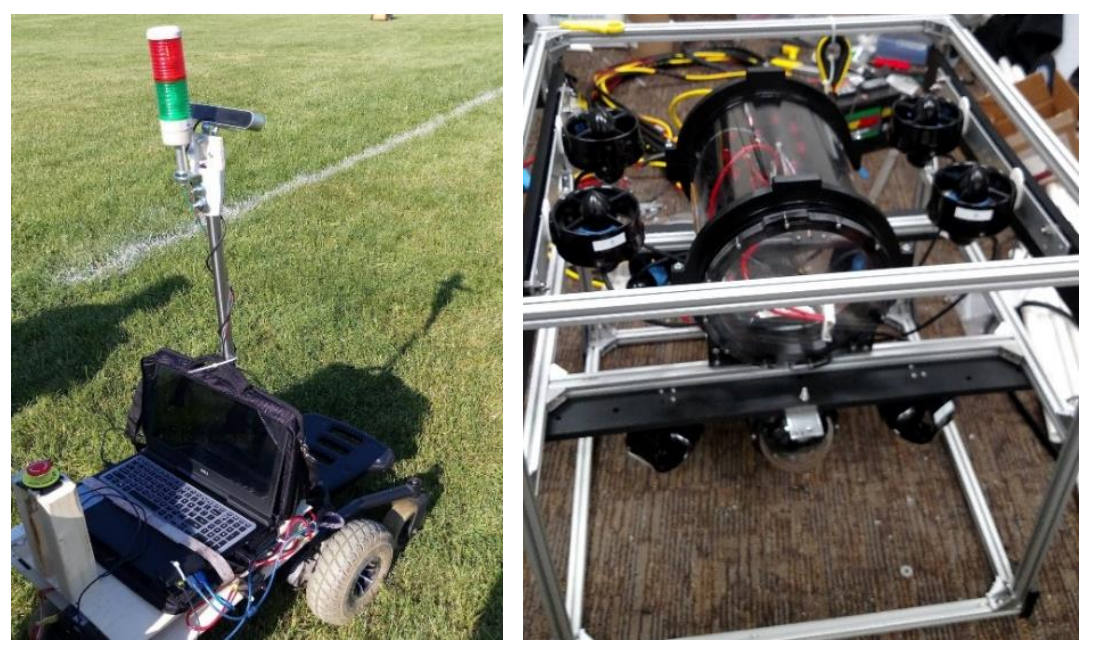

Figure 1. IGVC competition (left) vehicle and RoboSub competition vehicle (right). 
The robot the team designed for the 2018 competition is depicted in Figure 1 (on the left). It consisted of a modified electronic wheelchair frame, Zed depth sensing camera, GPS module, signal lights, and an emergency stop button. The vehicle is controlled via a laptop mounted on the back, which ran a student designed python application.

Another competition is RoboSub, an underwater robotics program in which teams design and build an Autonomous Underwater Vehicle (AUV). These vehicles must autonomously navigate through a series of tasks. The vehicle the team designed for this task has an eight-thruster setup, with four for vertical movement and four for horizontal movement. It also featured a Zed depth camera, which needed to be calibrated for use in underwater environments (due to light refraction). The RoboSub competition vehicle is also shown in Figure 1, on the right.

The learning experience that results from robotic competitions such as these will vary case by case. Although, the challenges involved in these competitions provide students with an opportunity to improve various technical and teamwork skills. In particular, robotics necessitate collaboration between students of different STEM fields, such as computer science, electrical engineering, and mechanical engineering (and related). Since students from these different fields need to collaborate with one another to find a solution, valuable teamwork skills may be gained as a result. In addition, the need to coordinate these activities in the group may increase leadership skills and experience for the individuals in those roles. Furthermore, these competitions can (in many instances) expose students to state of the art technology and potentially increase their chances of being hired in their desired field. Thus, participating in a competition may itself be a good item for a student to include on their resume. In addition, the competitions also provide a venue where potential employers may be scouting for talent. These questions are explored further in Section 5.

\section{Data and Analysis}

This section provides an overview of the data collection methods used for assessing this project. First the survey instrument is reviewed. Then data is presented and analyzed.

\subsection{Survey}

The surveys use questions from the Undergraduate Research Student Self-Assessment (URSSA) instrument [2] as well as questions initially designed to focus on particular activities and customized to focus on the specific values provided by robotic competition program participation.

These surveys (based on surveys previously implemented in [10]-[12]) were administered to multiple robotics teams with members with different demographic characteristics. Students are asked to identify benefits that they sought through participation and those they actually received. The participation benefit items in the survey include:

- Knowledge about the topic of the contest activities
- Knowledge about structured design processes 
- Knowledge about a particular technical topic

- Knowledge about project management

- Knowledge about time management

- Leadership experience

- Improving technical skills

- Improving time management skills

- Experience working with those from other disciplines

- Real-world project experience

- Item for resume

- Improved presentation skills

- Inclusion as author on technical paper

- Experience working on a large group project

- Experience with a structured design process
- Experience related to a particular technical topic

- Project management experience

- Time management experience

- Improving leadership skills

- Improving project management skills

- Understanding of how my discipline relates to others

- Learn other discipline's technical details/terminology

- Improved chance of being hired in desired field

- Increased self-confidence

- Ability to present at professional conference

- Recognition in the university community

Students are asked to identify pre- and post-participation status levels with regards to multiple metrics. They are also asked to rate the level of attribution of the change that they have indicated to program participation and to indicate whether they have participated in a number of specific activities typically associated with providing student benefit. The survey questions for these metrics include:

- I am interested in seeking employment in the field that I participated in

- I believe that participation will aid me in securing employment when graduating

- Technical skill in your area of focus before starting work on the project

- Level of comfort with the contest activities topic before starting work on the project

- Level of excitement with the contest activities topic before starting work on the project

- Level of presentation skills before starting work on the project

- Level of comfort with giving a presentation before starting work on the project

- Level of leadership skills before starting work on the project

- Level of leadership confidence before starting work on the project

- Level of project management skills before starting work on the project

- Level of project management confidence before starting work on the project

- Current technical skill in your area of focus

- Current level of comfort with the contest activities topic

- Current level of excitement with the contest activities topic

- Current level of presentation skills

- Current level of comfort with giving a presentation

- Current level of leadership skills

- Current level of leadership confidence

- Current level of project management skills

- Current level of project management confidence 
- Participation in this project has improved my technical skills:

- Participation in this project has improved my interest in the contest activities topic

- Participation in this project has improved my presentation skills

- Participation in this project has improved my comfort giving a presentation

- Participation in this project has improved my project management skills

- Participation in this project has improved my project management confidence

- Participation in this project has improved my leadership skills

- Participation in this project has improved my project leadership confidence

- Participation in this project has improved my time management skills

\subsection{Results}

The results from the survey are now presented. The benefits of participation that students seek compared to what they obtained is depicted in Figure 2. The values for participation benefit are Boolean (unscaled), such that boxes are checked to indicate whether a benefit is identified or not. The "seek" field represents the identified benefits being sought by a student before joining a project, and the "obtain" field represents the identified benefits that were obtained. 


\section{Participation Benefits}

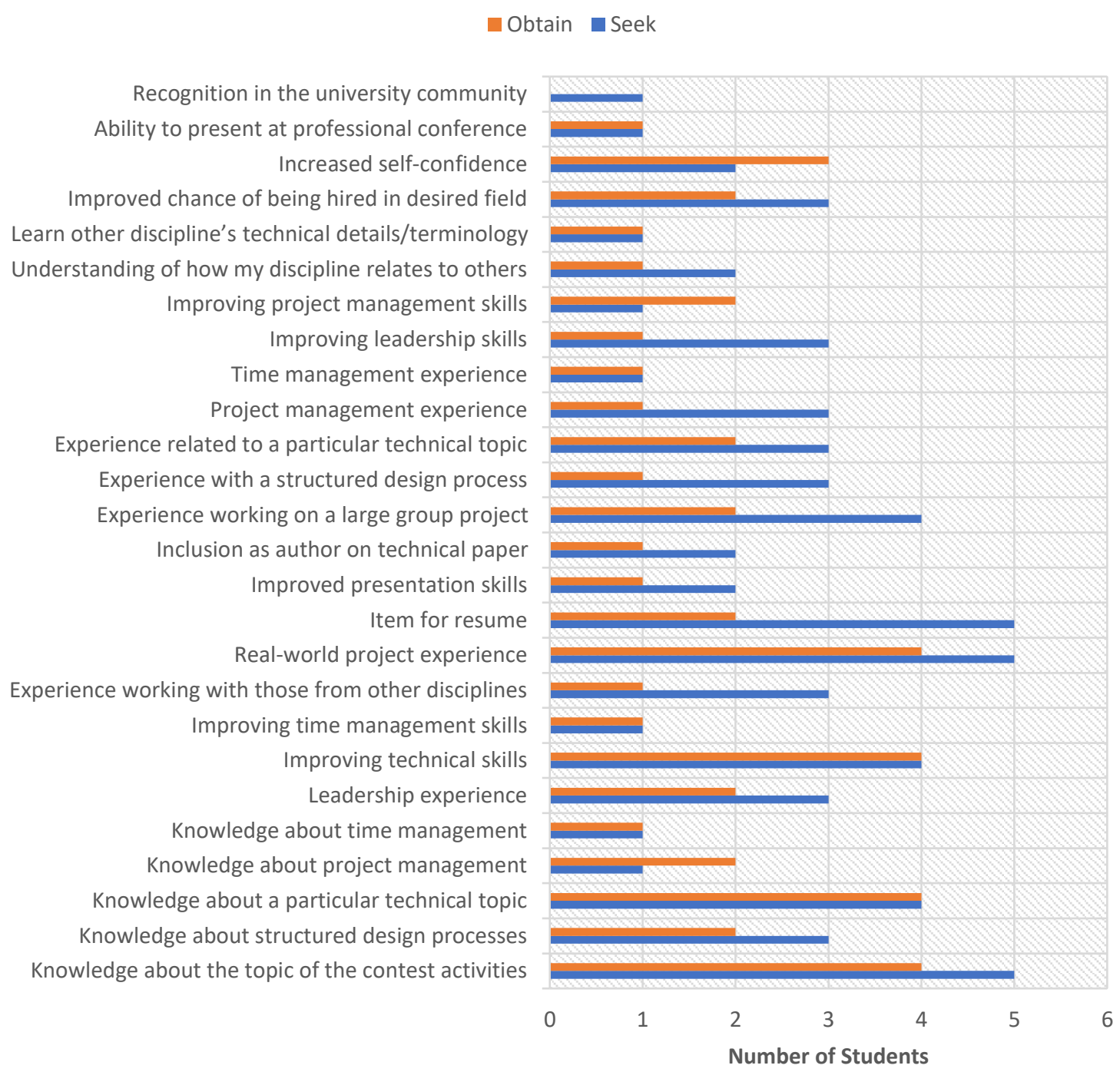

Figure 2. Graph depicting the participation benefits, both sought and obtained.

Students reason for participating is depicted in Figure 3. The values in this category are Boolean (unscaled). Multiple items can be selected by each student, such that responses are not limited to one item. 
Reason for participating
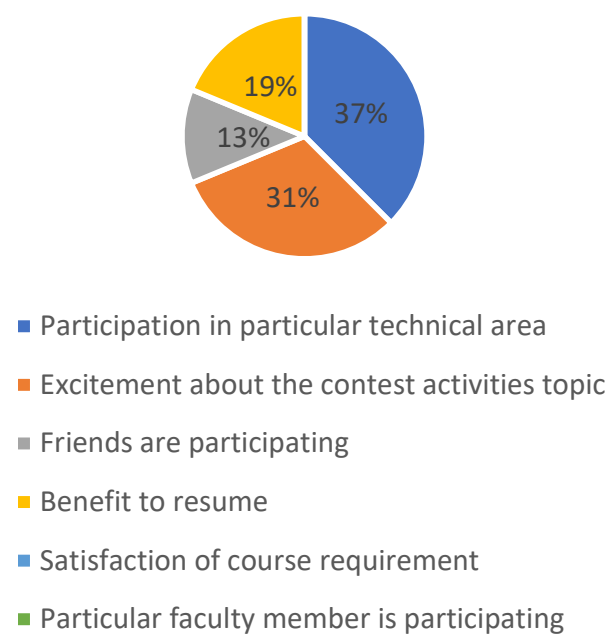

Figure 3. Pie chart depicting the reason for participating.

Participants also reported growth in technical skills, interest and various other skills. The results of survey questions related to these topics (all showing agreement with growth occurring in these areas due to program participation) are presented in Figures 4 to 8 .

\section{Technical Skills}

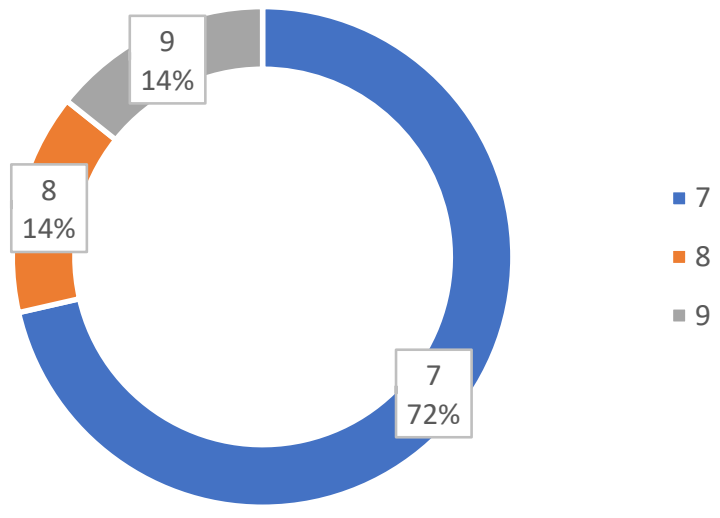

Figure 4. Graph depicting perceived improvement in technical skills (where no improvement corresponds to 1 , and strong improvement corresponds to 9). 


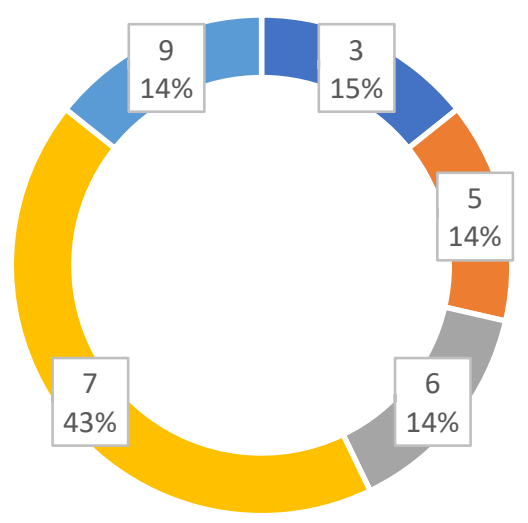

- 3

$\because 5$

$\because 6$

$\because 7$

$\because 9$

Figure 5. Graph showing the impact on the interest in the topics of the competition. Strong decrease corresponds to 1 , neutral corresponds to 5, and strong increase corresponds to 9 .

\section{Skill Level}

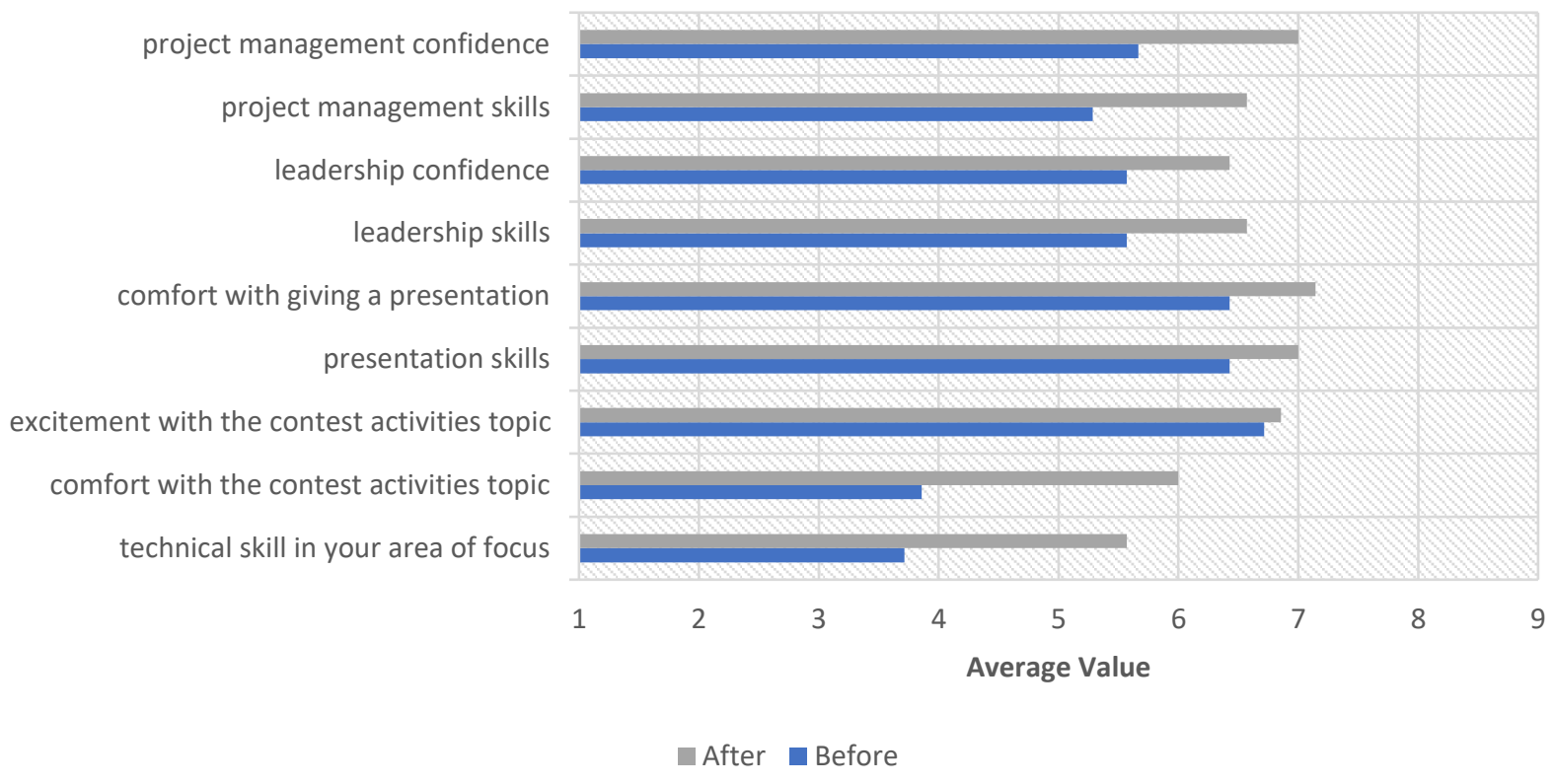

Figure 6. Graph showing the perceived change in skill level. Novice skill level corresponds to 1, intermediate skill level corresponds to 5, and expert skill level corresponds to 9. 


\section{Participation in this project has improved}

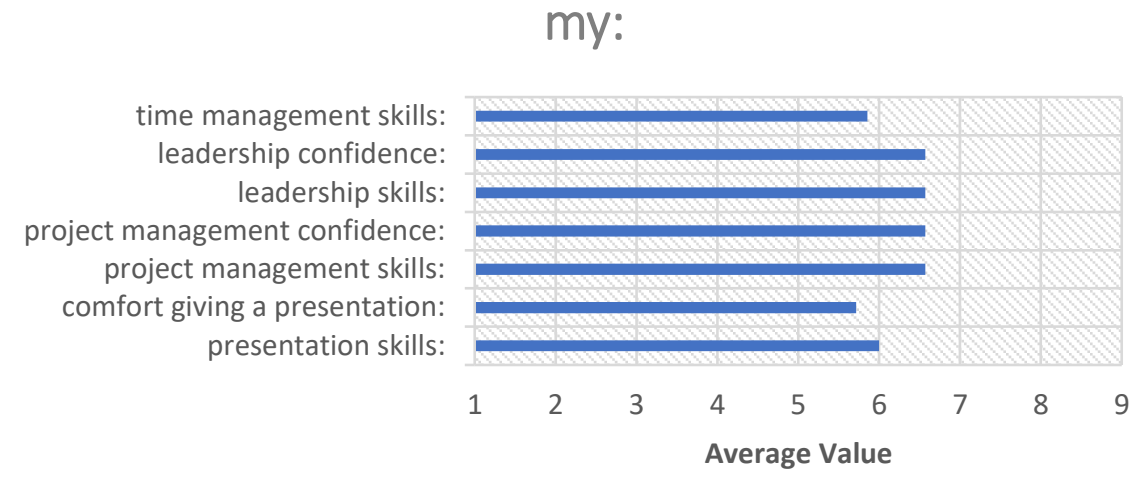

Figure 7. Perceived improvement in presentation, leadership, and management skills. Graph value is the average of scores, where strongly disagree corresponds to 1 , and strongly agree corresponds to 9.

\section{Employment}

I am interested in seeking employment in the field that I participated in:

I believe that participation will aid me in securing employment when graduating:

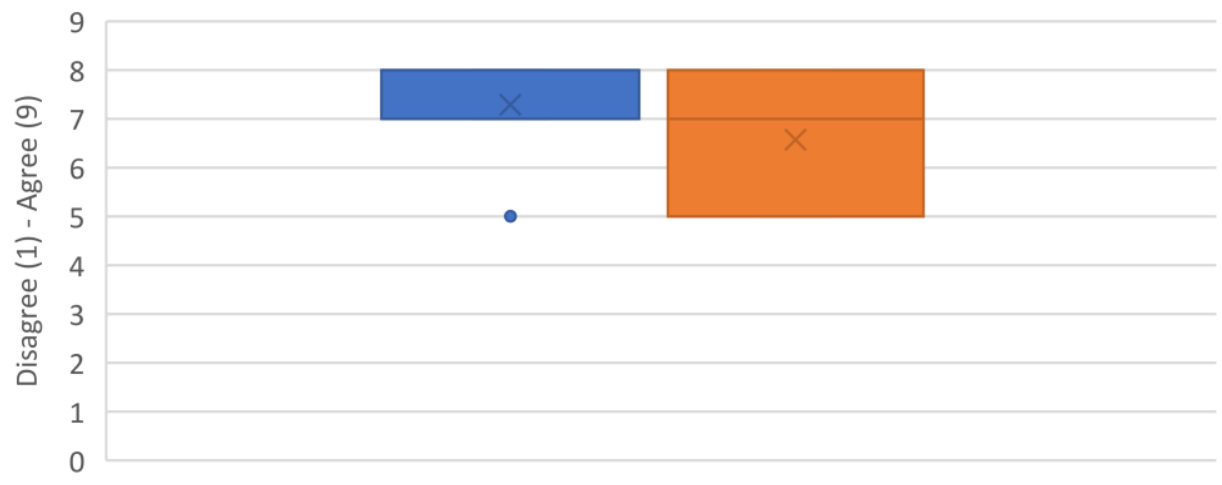

Figure 8. Chart depicting the seeking of employment in the desired field along with the perceived aid that the competition would provide in finding a job.

\section{Analysis of Educational Value}

While based on limited data, the forgoing demonstrates the significant value that students attain through robotic competition participation. Students recognize this, as demonstrated in the previous section, attributing gains in skills and comfort to their activities while involved with the program.

The identified benefits, in Figure 2, indicates that 'item for resume', and 'real-world project experience' categories are popular choices for what students are seeking. These particular 
categories may vary significantly depending on the robotics competition being considered. This may explain why the 'item for resume' category had less students claiming to have obtained it than 'real-world project experience.' Furthermore, the 'improved chance of being hired in desired field' had less students seeking and/or obtaining it. This lends to the notion that students view the benefits of these competitions as applying to their fields in general and aren't necessarily looking for a career in robotics. This is also evident in Figure 3, which has only twenty percent of students identifying that a resume item is a reason for joining the project. Although, the graph in Figure 8 provides further details to this and suggests that student participants wouldn't necessarily be opposed to a job in the robotics field.

The data in Figure 4 shows that all the surveyed students associate their participation with an increase in their technical skills. This is also evident in Figure 6, where the average technical skill level went up by two points. Moreover, the graph in Figure 2 shows that the students that sought increased technical skills all reported to have obtained technical skills as a result of their participation.

Another motivating factor for participating is the interest students have in the topics or narratives involved in the competitions. In Figure 3, roughly one third of students cite this as a reason for joining. In the graph depicted in Figure 5, the data suggests that, on average, interest in competition activities increases after participating. However, the data in Figure 6 suggests that this increase may be marginal. Although, the data does suggest that the comfort level with the competition activities increases after participating.

The graph in Figure 7 shows that, on average, students report that their leadership, presentation, and time management skills are increased due to participation. The time management skills may see an increase due to being exposed to a multi-semester project (depending on the competition) and having to constantly allocate time between school work and competition activities. Presentation skills also have an associated increase, and this may be due to the experience of defending certain design decisions when evaluated by the competition judges. Although, the word "presentation" may have an amount of ambiguity to it that could potentially skew results (i.e. does it refer to giving a speech, interacting with judges, or perhaps even the visual appeal of the robot). However, the graph in Figure 6 suggests that the perceived increase in presentation skills may be marginal. The increase in leadership skills may intuitively be increased for project leaders or subgroup leaders, but the increase noted by participants in general is slightly unexpected. It may be that having the different fields collaborating together creates a dynamic where participants can take the lead in their respective area of expertise. Although, this would depend on group size (among other factors), and further evaluation would be needed to draw any definite conclusions.

Lastly, most robotics competitions also provide an opportunity for students to meet with students who share similar interests at other schools and to work on robot construction (and repair) under significant pressure. They share the triumph of getting robots working (sometimes at the last moment and with limited parts and equipment to do so) and the satisfaction of completing the competition. Many contests have technical symposiums, in addition to the physical robot competitions, where students can present their work and receive feedback and questions from 
other teams, judges, other coaches and industry professionals. In short, robot competitions are a multi-faceted educational program and also a bonding experience for teams and between teams.

\section{Conclusions and Future Work}

This paper has presented initial work on the educational benefits of robotic competitions. It has reviewed relevant literature and discussed the work of several robotics teams at the North Dakota State University. Future work will involve conducting a more in-depth analysis of participation data and collecting data from additional participants over time to assess the long-term benefits of robotic program participation.

\section{References}

[1] J. Dias, K. Althoefer, and P. U. Lima, "Robot Competitions: What Did We Learn?," IEEE Robot. Autom. Mag., vol. 23, no. 1, pp. 16-18, 2016.

[2] A.-B. Hunter, T. J. Weston, S. L. Laursen, and H. Thiry, "URSSA: Evaluating Student Gains from Undergraduate Research in the Sciences," CUR Q., vol. 29, no. 3, pp. 15-19, 2009.

[3] A. Zdešar, S. Blažic, and G. Klančar, "Engineering Education in Wheeled Mobile Robotics," IFAC-PapersOnLine, vol. 50, no. 1, pp. 12173-12178, Jul. 2017.

[4] D. J. Ahlgren and I. M. Verner, "An International View of Robotics as an Educational Medium," in International Conference on Engineering Education, 2002.

[5] A. Merkouris, K. Chorianopoulos, and A. Kameas, "Teaching Programming in Secondary Education Through Embodied Computing Platforms," ACM Trans. Comput. Educ., vol. 17, no. 2, pp. 1-22, May 2017.

[6] G. A. Casan, E. Cervera, A. A. Moughlbay, J. Alemany, and P. Martinet, "ROS-based online robot programming for remote education and training," in 2015 IEEE International Conference on Robotics and Automation (ICRA), 2015, pp. 6101-6106.

[7] J. R. Savery, "Overview of Problem-based Learning : Definitions and Distinctions," Interdiscip. J. Probl. Learn., vol. 1, no. 1, pp. 9-20, 2006.

[8] C. E. Hmelo-Silver, "Problem-based learning: What and how do students learn?," Educational Psychology Review, vol. 16, no. 3. pp. 235-266, 2004.

[9] D. J. Ahlgren, I. M. Verner, D. Pack, and S. Richards, "Effective practices in robotics education," in ASEE Annual Conference Proceedings, 2004, pp. 4355-4369.

[10] J. Straub, R. Marsh, and D. Whalen, Small Spacecraft Development Project-Based Learning. New York, NY: Springer, 2017.

[11] J. Straub, R. Marsh, and D. Whalen, "Initial Results of the First NSF-Funded Research Experience for Undergraduates on Small Satellite Software," AIAA/USU Conf. Small Satell., 2015.

[12] J. Straub, "Initial results from the first national survey of student outcomes from small satellite program participation," in AIAA SPACE 2015 Conference and Exposition, 2015. 